\title{
Leucocyte-derived extracellular trap formation significantly contributes to Haemonchus contortus larval entrapment
}

Tamara Muñoz-Caro ${ }^{1 *}$, Mario C. Rubio R², Liliana M. R. Silva ${ }^{1}$, Gerd Magdowski ${ }^{3}$, Ulrich Gärtner ${ }^{3}$, Tom N. McNeilly , Anja Taubert ${ }^{1}$ and Carlos Hermosilla ${ }^{1}$

\begin{abstract}
Background: Polymorphonuclear neutrophil (PMN) and eosinophil extracellular trap (ETs) formation has recently been described as an important host effector mechanism against invading pathogens. So far, scarce evidence on metazoan-triggered ET formation has been published. We here describe for the first time Haemonchus contortus-triggered ETs being released by bovine PMN and ovine eosinophils in response to ensheathed and exsheathed third stage larvae $\left(L_{3}\right)$.

Methods: The visualization of ETs was achieved by SEM analysis. The identification of classical ETs components was performed via fluorescence microscopy analysis. The effect of larval exsheathment and parasite integrity on ET formation was evaluated via Pico Green ${ }^{\oplus}$ - fluorescence intensities. ETs formation under acidic conditions was assessed by using media of different $\mathrm{pH}$ ranges. Parasite entrapment was evaluated microscopically after co-culture of PMN and $\mathrm{L}_{3}$. ET inhibition experiments were performed using inhibitors against NADPH oxidase, NE and MPO. Eosinophil-derived ETs were estimated via fluorescence microscopy analysis.

Results: $L_{3}$ significantly induced PMN-mediated ETs and significant parasite entrapment through ETs structures was rapidly observed after 60 min of PMN and $\mathrm{L}_{3}$ co-culture. Co-localization studies of PMN-derived extracellular DNA with histones (H3), neutrophil elastase (NE) and myeloperoxidase (MPO) in parasite-entrapping structures confirmed the classical characteristics of ETs. Haemonchus contortus-triggered ETs were significantly diminished by NADPH oxidase-, NE- and MPO-inhibition. Interestingly, different forms of ETs, i.e. aggregated (aggETs), spread (sprETs) and diffused (diffETs) ETs, were induced by $\mathrm{L}_{3}$. AggETs and sprETs firmly ensnared larvae in a time dependent manner. Significantly stronger aggETs reactions were detected upon exposure of PMN to ensheathed larvae than to exsheathed ones. Low pH conditions as are present in the abomasum did not block ETosis and led to a moderate decrease of ETs. Eosinophil-ETs were identified extruding DNA via fluorescence staining.

Conclusion: We postulate that ETs may limit the establishment of $\mathrm{H}$. contortus within the definitive host by immobilizing the larvae and hampering them from migrating into the site of infection. Consequently, H. contortus-mediated ET formation might have an impact on the outcome of the disease. Finally, besides PMN-triggered ETs, we here present first indications of ETs being released by eosinophils upon $H$. contortus $L_{3}$ exposure.
\end{abstract}

Keywords: Haemonchus contortus, Neutrophil extracellular traps, Innate immunity, ETosis

\footnotetext{
* Correspondence: Tamara.Munoz-Caro@vetmed.uni-giessen.de; mariocesarrubio@hotmail.com

${ }^{1}$ Institute of Parasitology, Justus Liebig University Giessen, Giessen, Germany

${ }^{2}$ Faculty of Veterinary Medicine and Zootechny, Autonomous University of

Sinaloa, Culiacán, Mexico

Full list of author information is available at the end of the article
} 


\section{Background}

Haemonchus contortus is a gastrointestinal nematode with worldwide distribution. This abomasal parasite leads to significant economic losses particularly in small ruminant livestock [1]. Haemonchosis is acquired by ingesting infective third-stage larvae $\left(\mathrm{L}_{3}\right)$ from contaminated pasture. After exsheathment, $\mathrm{L}_{3}$ penetrates the abomasal glands where it develops to fourth-stage larvae $\left(\mathrm{L}_{4}\right)$ and thereafter to dioecious haematophagous preadult larvae $\left(\mathrm{L}_{5}\right)$ and adults. Adult $H$. contortus nematodes are found in the lumen of the abomasum in herbivores [2-5]; or the stomach of omnivores [6] consuming up to $0.05 \mathrm{ml}$ host blood per worm daily [7], which results in hemorrhagic abomasitis (gastritis), anaemia, oedema and associated complications often leading to death of severely infected animals [8].

$H$. contortus infections in ruminants are known to elicit a Th2 type-dominated host immune response being characterized by the recruitment of large numbers of eosinophils, mast cells and globule leucocytes and to the production of locally active and circulating antibodies [9-11]. Nonetheless, little is known on the very early host innate immune responses against $H$. contortus. In this scenario, the relative inaccessibility of infective $H$. contortus $\mathrm{L}_{3}$ within abomasal/gastric glands for host leucocytes poses unique challenges to the innate immune system, which has evolved several specialized strategies for parasite control [12]. Parasite colonization of the host abomasum initially depends on the motility of the larvae and the parasite load. Thus, some host individuals, after sensitization via previous infections, can modify the microenvironmental conditions of this niche to expel the parasite [13]. There is evidence showing that helminths activate the alternative complement pathway binding opsonins on their surface [14]. Moreover, within the innate immune response polymorphonuclear neutrophils (PMN; [15]) and eosinophils are considered as fundamental leucocytes forming the first line of defense against metazoan nematodes and the first leucocytes to be recruited to the site of infection [16-19]. Various authors have reported that eosinophils are capable of immobilizing infective larvae of diverse species of nematodes in vitro and in vivo $[9,10,20,21]$. Furthermore, incubation of $H$. contortus $\mathrm{L}_{3}$ with antibodies raised against $\mathrm{HcsL}_{3}$ antigens in the presence of ovine eosinophils resulted in significant larval killing after 24 h [20]. In addition, it has been demonstrated that eosinophils are essentially involved in the expulsion of diverse nematodes in vivo, such as Strongyloides stercoralis [22], Onchocerca lienalis [23], Trichinella spiralis [24] and Trichostrongylus colubriformis [25]. Alongside phagocytosis and oxidative burst, leucocytes are capable of triggering extracellular traps (ETs) as a novel effector mechanism. This results in the cellular release of granule proteins and chromatin upon activation that together form extracellular fibers capable of binding and killing Gram-positive and -negative bacteria and parasites $[16,26]$. So far, the mechanism of ET formation has been attributed to PMN [16], mast cells [27], macrophages [28], eosinophils [29] and monocytes [30, 31] and thus appears to be a general effector mechanism of innate immune cells. Most studies on pathogen-triggered ETs have been focused on bacterial, viral and fungal infections $[17,32-34]$. However, little attention has been paid to parasites as ET-inducers [26] and studies of ET induction by parasites have mainly focused on protozoans [35-40]. So far, only two helminth species, i.e. Schistosoma japonicum [41] and Strongyloides stercolaris [15], have been proven to induce NETs.

With the present work we add a new species to the group of metazoan-ET-inducers and highlight the capability of ETs to entrap this large parasite. The current data suggest $H$. contortus-mediated NET formation may influence the outcome of the infection in affected animals in vivo. Furthermore, we here provide first evidence on eosinophil-derived ET formation in response to a helminth parasite.

\section{Methods}

\section{Ethic statement}

All animal procedures were performed according to the Moredun Research Institute regulations and to the Justus Liebig University Animal Care Committee guidelines, approved by the Ethic Commission for Experimental Animal Studies of the State of Hesse and in accordance to the current European Animal Welfare Legislation: ART13TFEU.

\section{Animals and parasites}

Three male Merino sheep were purchased from a local farmer at the age of five months, treated with a single dose of toltrazuril $\left[20 \mathrm{mg} / \mathrm{kg}\right.$ body weight (bw), Baycox ${ }^{\oplus}$ $5 \%$; Bayer Animal Health] and benzimidazole $(10 \mathrm{mg} / \mathrm{kg}$ bw, Panacur; Bayer Animal Health). The sheep were controlled for parasitic infections via weekly coprological analyses and, when deemed parasite free, maintained under parasite-free conditions within a large animal stable equipped with a laminar flow lock entrance until experimental infection (Institute of Parasitology, Justus Liebig University, Giessen, Germany). Animals were fed with commercial starter pellet concentrates (Lämmerpellets ${ }^{\oplus}$, Deuka). Water and sterilized hay were given ad libitum.

Animals were infected per os with $8 \times 10^{3}$ viable ensheathed $H$. contortus $\mathrm{L}_{3}$ (in house strain) suspended in tap water. Following prepatency of approximately three weeks, cotton faecal collection bags were fixed to the anuses of sheep to collect faeces and were emptied each day. 
The isolation of excreted $H$. contortus eggs and exogenous in vitro culture into third stage larvae were performed as previously described elsewhere [42]. Faecal samples (10-50 g) were transferred to a jar and mixed with commercially purchased sawdust until a crumbly consistency was obtained, and, if necessary, dampened with tap water. Thereafter, the jars were capped and incubated at $27-28{ }^{\circ} \mathrm{C}$ for $7-8$ days. After incubation, tap water was added to the culture until the jar was filled up to the brim, the jar was turned upside down on a petri dish. Then, 10-20 $\mathrm{ml}$ of tap water was added into the petri dish and the jars were incubated overnight at room temperature (RT). Thereafter, the fluid containing $H$. contortus ensheathed $\mathrm{L}_{3}$ was collected, transferred to a conical tube (Greiner) and the $\mathrm{L}_{3}$ were sedimented at unit gravity (at least $30 \mathrm{~min}, \mathrm{RT}$ ). Afterwards, the supernatant was discarded; the $\mathrm{L}_{3}$ of $H$. contortus were counted, suspended in sterile PBS and stored at $4{ }^{\circ} \mathrm{C}$ until further use. $\mathrm{L}_{3}$-related ET experiments were performed within 4 weeks after parasite collection in order to prevent morphological alterations or death of the larvae.

\section{Exsheathment of Haemonchus contortus $\mathrm{L}_{3}$}

For exsheathment, vital ensheathed $\mathrm{L}_{3}$ (1500 larvae in $5 \mathrm{ml}$ tap water) were exposed to sodium hypochlorite solution $(0.3 \% v / v ; 5 \mathrm{~min}, \mathrm{RT}$, Merck). Active larval exsheathment was observed under an inverted DMIRB ${ }^{\circ}$ microscope (Leica). When at least $80 \%$ of the $H$. contortus $\mathrm{L}_{3}$ had exsheathed, the larvae were washed in sterile PBS $(400 \times g, 3 \times 5 \mathrm{~min})$ and kept at $4{ }^{\circ} \mathrm{C}$ for further use within the same day.

\section{Isolation of bovine PMN and ovine eosinophils}

For PMN isolation, healthy adult female Holstein-Frisian cows $(n=3)$ were bled by puncture of the jugular vein. Heparinized blood was diluted under sterile conditions in an equal volume of sterile phosphate buffered saline (PBS) containing $0.02 \%$ EDTA (Sigma-Aldrich). The mixture was layered on Biocoll ${ }^{\bullet}$ separating solution (Biochrom AG) and centrifuged at $800 \times g$ for $45 \mathrm{~min}$. After removal of plasma, lymphocytes and monocytes in the upper layers of the gradient, the cells were re-suspended in $25 \mathrm{ml}$ sterile distilled water to lyse erythrocytes. Osmolarity was immediately readjusted by adding $10 \mathrm{x}$ sterile Hank's Balanced Salt Solution (HBSS, Biochrom AG) and the cells were pelleted $(400 \times g, 10 \mathrm{~min})$. Thereafter, the cells were washed twice $\left(400 \times g, 10 \mathrm{~min}, 4^{\circ} \mathrm{C}\right)$ in complete RPMI 1640 medium without phenol red (Sigma-Aldrich). The cells were counted in a Neubauer haemocytometer chamber and incubated at $37{ }^{\circ} \mathrm{C}$ and $5 \% \mathrm{CO}_{2}$ atmosphere for at least $30 \mathrm{~min}$ before use. The PMN-enriched samples had $>90 \%$ purity.

For ovine eosinophil isolation, heparinized blood $(25 \mathrm{ml})$ was collected from Texel-cross lambs $(n=3)$, with patent $H$. contortus infection, diluted 1:2 with sterile PBS- containing $0.05 \%$ EDTA (Sigma-Aldrich) and layered on top of a $70 \%$ Percoll gradient $(25 \mathrm{ml}$, SigmaAldrich). The sample was centrifuged (20 min, $400 \times g$, $4{ }^{\circ} \mathrm{C}$ ). Within the gradient, the eosinophil-enriched layer appeared in between the peripheral blood mononuclear cell- (PBMC) and the PMN-rich layer. Eosinophils were withdrawn by aspiration in a sterile Pasteur pipette, resuspended in $25 \mathrm{ml}$ PBS-EDTA and washed in PBS $\left(400 \times g, 2 \times 5 \mathrm{~min}, 4^{\circ} \mathrm{C}\right)$. The cells were re-suspended in culture medium [RPMI 1640, $10 \%$ heat inactivated foetal calf serum (hiFCS), $2 \mathrm{mM} \mathrm{L}$-glutamine, $100 \mathrm{U} / \mathrm{ml}$ penicillin, $100 \mu \mathrm{g} / \mathrm{ml}$ streptomycin and $50 \mu \mathrm{M} 2$ mercaptoethanol, all Sigma-Aldrich]. Eosinophil purity was determined by light microscopy of cells stained with Diff-Quick and haematoxylin-eosin staining after cytocentrifugation onto glass slides. Eosinophil counting was performed in a Neubauer haemocytometer chamber. Eosinophils were incubated at $37{ }^{\circ} \mathrm{C}$ and $5 \% \mathrm{CO}_{2}$ atmosphere for at least $30 \mathrm{~min}$ before use. The viability of eosinophils was tested by using the trypan blue exclusion test (Sigma-Aldrich). Eosinophil-enriched samples had $30 \%$ eosinophils.

\section{Scanning electron microscopy (SEM)}

Bovine PMN $\left(n=3,5 \times 10^{5}\right)$ were co-cultured with vital ensheathed $\mathrm{L}_{3}$ of $H$. contortus (100 larvae/sample) on poly-L-lysine (Sigma-Aldrich) pre-coated coverslips $\left(60 \mathrm{~min}, 37^{\circ} \mathrm{C}\right)$. After incubation, the samples were fixed in $2.5 \%$ glutaraldehyde (60 min, RT, Merck), post-fixed in $1 \%$ osmium tetroxide (Merck), washed in distilled water, dehydrated, critical point dried by $\mathrm{CO}_{2}$-treatment and spayed with gold. Thereafter, samples were examined with a Philips XL30 scanning electron microscope at the Institute of Anatomy and Cell Biology, Justus Liebig University Giessen, Germany.

\section{Visualization of NETs and detection of histones, neutrophil elastase (NE) and myeloperoxidase (MPO) in Haemonchus contortus-induced NETs}

Bovine PMN $\left(n=3,5 \times 10^{5}\right)$ were co-cultured with vital ensheathed $H$. contortus $\mathrm{L}_{3}$ (100 larvae/sample) on poly-L-lysine-treated coverslips $\left(60 \mathrm{~min}, 37^{\circ} \mathrm{C}\right)$. Thereafter, the samples were fixed (overnight, $4 \%$ paraformaldehyde, on ice, Merck) and stored at $4{ }^{\circ} \mathrm{C}$ until further use. The experiments were performed in duplicates. In each set of data, 2 coverslips were used per condition and the entire coverslip was further analysed. NET structures were visualized by staining extracellular nucleic acid with Sytox Orange ${ }^{ø}$ as previously recorded [43-45]. For the detection of histones, MPO and NE within NET structures the following specific antibodies were used: anti-histone $\mathrm{H} 1$, $\mathrm{H} 2 \mathrm{~A} / \mathrm{H} 2 \mathrm{~B}, \mathrm{H} 3, \mathrm{H} 4$ monoclonal antibody [mouse clone H11-4, 1:1000, 1 h, RT, Merck Millipore], anti-MPO 
antibodies (rabbit polyclonal anti-MPO, Alexa Fluor 488, 1:200, 24 h, RT, ABIN906866, antibodies-online.com) and anti-NE antibodies (rabbit polyclonal anti human NE, 1:200, 24 h, RT, AB68672, Abcam). Prior to antibody exposure, the samples were blocked with bovine serum albumin (30 min, BSA $2 \%$, Sigma-Aldrich). Following antibody exposure, the samples were washed twice with PBS and incubated in respective secondary antibody solutions [Alexa Fluor 488 goat anti-mouse IgG and Alexa Fluor 488 goat anti-rabbit IgG (both from Life Technologies) $30 \mathrm{~min}, 1: 500, \mathrm{RT}]$. Finally, the samples were stained with Sytox Orange ${ }^{\bullet}$ (S-11368, Invitrogen, 1:1000, $15 \mathrm{~min}$ in the dark), washed with PBS and mounted in anti-fading buffer (Mowiol'; Sigma-Aldrich). The visualization of NETs based on co-localized extracellular DNA staining and histone-, MPO- and NE-derived signals was achieved by using an inverted Olympus IX $81^{\circ}$ fluorescence microscope.

\section{Visualization of eosinophil-derived Haemonchus contortus-induced ETs}

Co-culture of ovine eosinophils $\left(n=3,1 \times 10^{6}\right)$ with either ensheathed or exsheathed $H$. contortus $\mathrm{L}_{3}$ (150 larvae/sample each) was carried out on poly- ${ }_{-}-$lysinetreated coverslips $\left(60 \mathrm{~min}, 37^{\circ} \mathrm{C}\right)$. After the incubation period, the samples were fixed (overnight, $4 \%$ paraformaldehyde, on ice). Eosinophilic ET structures were visualized by staining extracellular DNA with Pico Green ${ }^{\circ}$ (Life Technologies, 1:200 diluted in $10 \mathrm{mM}$ Tris/1 mM EDTA) according to [45]. Moreover, eosinophilic granules were labelled with alcoholic Eosin $\mathrm{Y}^{\circ}$ solution $(10 \mathrm{~s}$, RT, Sigma-Aldrich) and thereafter washed twice with sterile PBS. The cells were then mounted in ProLong ${ }^{\circ}$ anti-fading reagents (Life Technologies). The visualization of eosinophil-derived ETs was achieved using an inverted Olympus IX $8^{\circledR}$ phase contrast/fluorescence microscope.

\section{Quantification of Haemonchus contortus-induced NETs} Bovine PMN $\left(n=3 ; 5 \times 10^{5}\right.$ cells $\left./ 200 \mu \mathrm{l}\right)$ were exposed either to vital ensheathed larvae (40 larvae/sample) or heat-inactivated $\left(60^{\circ} \mathrm{C}, 60 \mathrm{~min}\right.$ ) larvae (40 larvae/sample) in RPMI 1640 medium (1 \% PS, without phenol red) for $60 \mathrm{~min}$ at $37{ }^{\circ} \mathrm{C}$ in 96 -well flat-bottom plates (Nunc). Thereafter, micrococcal nuclease was added (5 U/well, New England Biolabs) and incubated for $15 \mathrm{~min}$ at $37^{\circ} \mathrm{C}$. Then the samples were centrifuged $(250 \times g, 5 \mathrm{~min})$ and the supernatants $(100 \mu \mathrm{l} /$ well $)$ were transferred to a 96 well flat-bottom plate (Nunc). The samples were stained by Pico Green ${ }^{\circ}(50 \mu \mathrm{l} /$ well, 1:200 in $10 \mathrm{mM}$ Tris/ $1 \mathrm{mM}$ EDTA) and NET formation was quantified by spectrofluorometric analysis at an excitation wavelength of $484 \mathrm{~nm}$ and emission wavelength $520 \mathrm{~nm}$ using an automated plate monochrome reader (Varioskan Flash'; Thermo Scientific). For negative controls, PMN in plain medium were used. Stimulation of PMN with zymosan $(1 \mathrm{mg} / \mathrm{ml}$,
Invitrogen) served as positive control. For the quantification of the different types of NETs, bovine PMN $(n=3$, $5 \times 10^{5}$ ) were seeded on poly-L-lysine pre-coated coverslips and exposed to $H$. contortus $\mathrm{L}_{3}$ (20 larvae/sample) in $300 \mu \mathrm{l}$ RPMI medium (1 \% PS, without phenol red) for $30 \mathrm{~min}$ and $1 \mathrm{~h}$ at $37^{\circ} \mathrm{C}$. Thereafter NET formation was detected via fluorescence microscopy analysis using an anti-histone $\mathrm{H} 1, \mathrm{H} 2 \mathrm{~A} / \mathrm{H} 2 \mathrm{~B}, \mathrm{H} 3, \mathrm{H} 4$ monoclonal antibody [mouse clone H11-4, 1:1000, $1 \mathrm{~h}, \mathrm{RT}$ ] jointly with a secondary antibody (Alexa Fluor 488 goat anti-mouse IgG). Moreover, for staining extracellular DNA, Sytox Orange $^{\circ}$ (S-11368, Invitrogen, 1:1000, $15 \mathrm{~min}$ in the dark) was used.

\section{Entrapment assay of Haemoncus contortus $\mathrm{L}_{3}$}

Bovine PMN $\left(n=3,5 \times 10^{5}\right)$ were seeded on poly-L-lysine pre-coated coverslips and exposed to ensheathed $H$. contortus $\mathrm{L}_{3}$ (100 larvae/sample) in $300 \mu \mathrm{l}$ RPMI medium (1\% PS, without phenol red, Sigma-Aldrich) for 30 and $60 \mathrm{~min}$ at $37{ }^{\circ} \mathrm{C}$. Thereafter, the coverslips were fixed $(4 \%$ paraformaldehyde, overnight on ice) and PMN-entrapped larvae were counted by using an inverted $\mathrm{DMIRB}^{\circ}$ phasecontrast microscope (Leica). Larvae were considered as entrapped when aggNET or sprNET were in contact with the larvae. The data are expressed as percentage of entrapped $\mathrm{L}_{3}$ relative to the total amount of $H$. contortus $\mathrm{L}_{3}$.

\section{NET-associated larvicidal assay}

Bovine PMN $\left(n=3,5 \times 10^{5}\right)$ were seeded on poly-L-lysine pre-coated coverslips and exposed to ensheathed $H$. contortus $\mathrm{L}_{3}$ (50 larvae/sample) in $300 \mu \mathrm{l}$ RPMI medium ( $1 \%$ PS, without phenol red) for 24,36 and $48 \mathrm{~h}$ at $37{ }^{\circ} \mathrm{C}$. Thereafter, DNase I (Sigma Aldrich) was added to the coverslips to resolve NET structures and larval survival was determined microscopically based on the presence or absence of larval motility. In addition, parasite viability was evaluated by using the trypan blue exclusion test (1:10 dilution, Sigma-Aldrich). For reference samples, the equal number of non-PMN-exposed larvae was used.

\section{Identification and quantification of aggregated NETs (aggNETs) induced by Haemonchus contortus $\mathrm{L}_{3}$}

Bovine PMN $\left(n=3,5 \times 10^{5}\right)$ were seeded on poly-L-lysine pre-coated coverslips and exposed to both, ensheathed and exsheathed $H$. contortus $\mathrm{L}_{3}$ (50 larvae/sample) in $300 \mu \mathrm{l}$ RPMI medium (1 \% PS, without phenol red) for $1 \mathrm{~h}$ at $37{ }^{\circ} \mathrm{C}$. Thereafter, the samples were fixed $(4 \%$ paraformaldehyde, overnight, on ice). Each sample was analysed microscopically using an inverted phase-contrast microscope (DMIRB ${ }^{\circ}$, Leica). AggNETs were defined as clusters of NET-like structures with a "ball of yarn" morphology (see Fig. 2f red arrow) being larger than $50 \mu \mathrm{m}$ in diameter. Within each sample, all structures with the described characteristics were counted. For negative controls, PMN 
in plain medium was used. Stimulation of PMN with zymosan $(1 \mathrm{mg} / \mathrm{ml})$ served as positive controls as described elsewhere [45].

\section{NET inhibition assays}

For NET inhibition assays the following inhibitors were used according to [46] and [39]: the NADPH oxidase inhibitor diphenylene iodonium (DPI, $10 \mu \mathrm{M}$, SigmaAldrich), the MPO inhibitor 4-Aminobenzoic acid hydrazide (ABAH, $100 \mu \mathrm{M}$, Calbiochem) and the NE inhibitor Suc-Ala-Ala-Pro-Val chloromethyl ketone (CMK, $1 \mathrm{mM}$, Sigma-Aldrich). PMN $\left(n=3,5 \times 10^{5}\right)$ were preincubated with respective inhibitors $\left(30 \mathrm{~min}, 37^{\circ} \mathrm{C}, 5 \%\right.$ $\mathrm{CO}_{2}$ ) prior to exposure to vital ensheathed $\mathrm{L}_{3}$ (80 larvae/ sample, $60 \mathrm{~min}$ ). For no-inhibitor controls, non-treated PMN were exposed to larvae. For negative controls, PMN in plain medium were used. Stimulation of PMN with zymosan $(1 \mathrm{mg} / \mathrm{ml})$ served as positive control. NET formation was quantified as described above.

Haemonchus contortus-induced NETs under acidic conditions In order to verify whether NET formation occurs in abomasal acidic $\mathrm{pH}$ conditions, the cell culture medium (RPMI 1640) was adjusted to different acidic pH conditions: 2.0, 2.5, 3.0 and 3.5 prior to NET assays. For controls a $\mathrm{pH}$ of 7.0 was applied. Bovine PMN $(n=3$, $5 \times 10^{5}$ ) were seeded on poly-L-lysine pre-coated coverslips and exposed to $H$. contortus $\mathrm{L}_{3}$ (20 larvae/sample) in $300 \mu \mathrm{l}$ RPMI medium (1 \% PS, without phenol red) for $1 \mathrm{~h}$ at $37{ }^{\circ} \mathrm{C}$. Thereafter NET formation was detected using a fluorescence microscope as previously described using an anti-histone $\mathrm{H} 1, \mathrm{H} 2 \mathrm{~A} / \mathrm{H} 2 \mathrm{~B}, \mathrm{H} 3, \mathrm{H} 4$ monoclonal antibody [mouse clone H11-4, 1:1000, 1 h, RT] jointly with a secondary antibody (Alexa Fluor ${ }^{\oplus} 488$ goat antimouse IgG). Moreover, for staining extracellular DNA, Sytox Orange $^{\oplus}$ (S-11368, Invitrogen, 1:1000, $15 \mathrm{~min}$ in the dark) was used.

\section{Statistical analysis}

By using normal distribution of the data, co-culture/stimulation conditions were compared by one- or two-factorial analyses of variance (ANOVA) with repeated measures. Differences were regarded as significant at a level of $p \leq 0.05$ and analysed by GraphPad Prism ${ }^{\odot}$ programme.

\section{Results}

\section{Haemonchus contortus $\mathrm{L}_{3}$ induce different types of NETs}

Scanning electron microscopy (SEM) analyses revealed that exposure of bovine PMN to ensheathed third-stage larvae of $H$. contortus triggers the formation of morphologically different NETs. Typically "diffuse" NETs (diffNETs) are composed of a complex of extracellular decondensed chromatin decorated with antimicrobial proteins with globular and compact form with a size of
25-28 nm diameter. Moreover, "spread" NETs (sprNETs) were observed consisting of smooth and elongated weblike structures of decondensed chromatin and antimicrobial proteins composed exclusively by thin fibers with a diameter of 15-17 nm (Fig. 1a, b). Furthermore, a marked presence of so-called "aggregated" NETs (aggNETs), according to [47] were displayed as large clusters of NET-like structures with a "ball of yarn"-like clumpy and massive appearance involving a high number of PMN. These conglomerates of NETs were composed by extracellular chromatin studded with phagocyte-granular proteins with sizes larger than $50 \mu \mathrm{m}$ in diameter. SprNETs and aggNET formation led to larval entrapment and, in some cases of aggNETs, to an almost entire coverage of the larvae (Fig. 1c, d). Overall, both forms of NETs, i.e. sprNET and aggNET, appeared capable of firmly ensnaring the larvae (see Additional files 1, 2: movies 1 and 2). Furthermore, we also observed a significant reduction in larval forward-motility due to an anchor-like effect of fine NET structures (sprNETs), which are connected to the aggNETs entrapping larvae and clearly hampering larval motility (see Additional files 1, 2: movies 1 and 2).

Fluorescence-based analyses confirmed the classical components of NET formation. As such, staining by Sytox Orange ${ }^{\odot}$ (Fig. 2a, d, g) proved the DNA nature of extracellular NET-like structures being formed by PMN after $H$. contortus L3 exposure. In addition, MPO-, NEand histone- $(\mathrm{H} 1, \mathrm{H} 2 \mathrm{~A} / \mathrm{H} 2 \mathrm{~B}, \mathrm{H} 3$ and $\mathrm{H} 4)$ positive signals were detected in co-localization with DNA-positive NET structures (Fig. 2c, f, i). Interestingly, we also observed different forms of NETs, i.e., sprNETs (Fig. 2c, f, white arrows), aggNETs (Fig. 2f, red arrows), diffused NETs (diffNET, Fig. 2f, yellow arrow) and clasp-like NET formation (Fig. 2 i, red arrow) sticking mainly to the anterior part of the larvae, consistent with observations made by SEM analyses (see Fig. 1).

Quantitative analyses on total NET formation showed that ensheathed and exsheathed $\mathrm{L}_{3}$ significantly induced NETosis in bovine PMN ( $p \leq 0.05$, Fig. 3a). As expected, this reaction was significantly and almost entirely resolved by DNase I treatment in both, exsheathed and ensheathed larvae (larvae vs. DNase I: $p \leq 0.05$, Fig. 3a). Furthermore, quantitative assessment of aggNETs revealed that exposure of PMN to ensheathed $\mathrm{L}_{3}$ significantly induced aggNET in bovine PMN when compared to negative controls ( $p \leq 0.01$, Fig. 3b). Comparative approaches revealed that ensheathed larvae triggered NETosis significantly more than exsheathed ones ( $p \leq 0.05$, Fig $3 b)$, indicating that surface cuticle components are able to effectively trigger NETosis. Furthermore, analysis of the frequency of different NET types induced by $H$. contortus was performed for different time spans (30 and $60 \mathrm{~min}$ ). As outcomes of this experiment, at both time points the three different types of NETs were observed to be significantly 

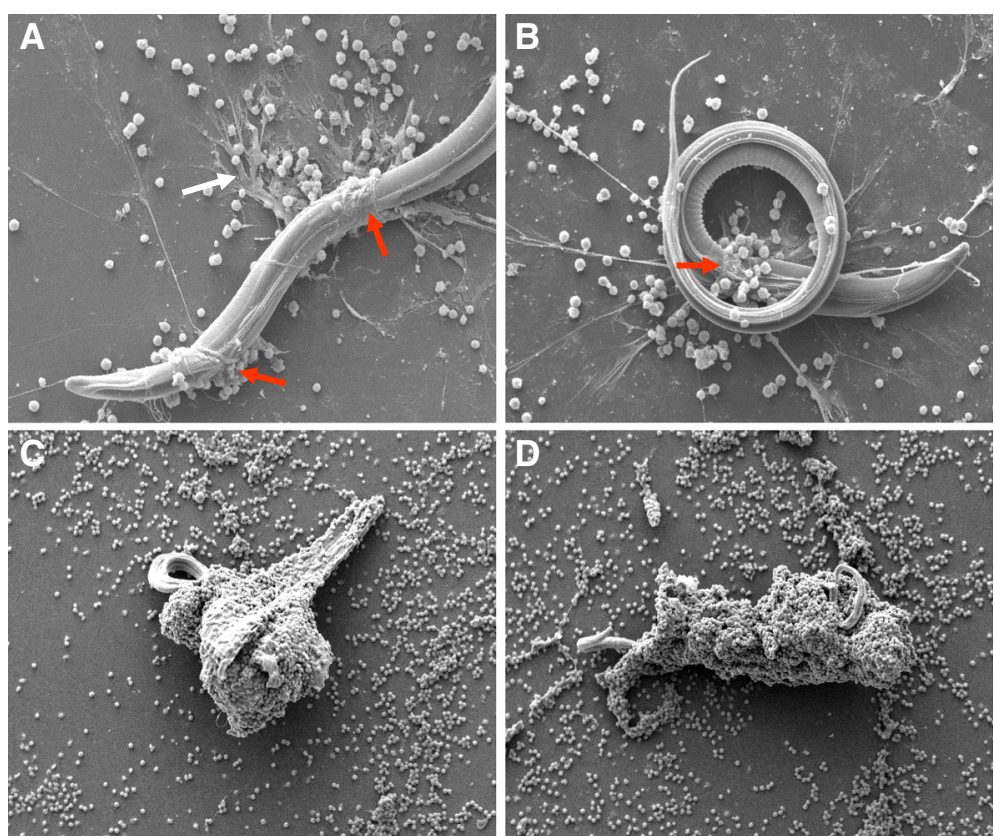

Fig. 1 Co-culture of Haemonchus contortus larvae and bovine PMN triggers NET formation. Scanning electron microscopy (SEM) analysis revealed different forms of NET formation induced by $\mathrm{H}$. contortus $\mathrm{L}_{3}$ in bovine PMN contributing to larval immobilization. $\mathbf{a}, \mathbf{b}$ : $H$. contortus trigger spread NET formation (sprNET) $\mathbf{c}$, d: H. contortus trigger aggregated NET formation (aggNET)
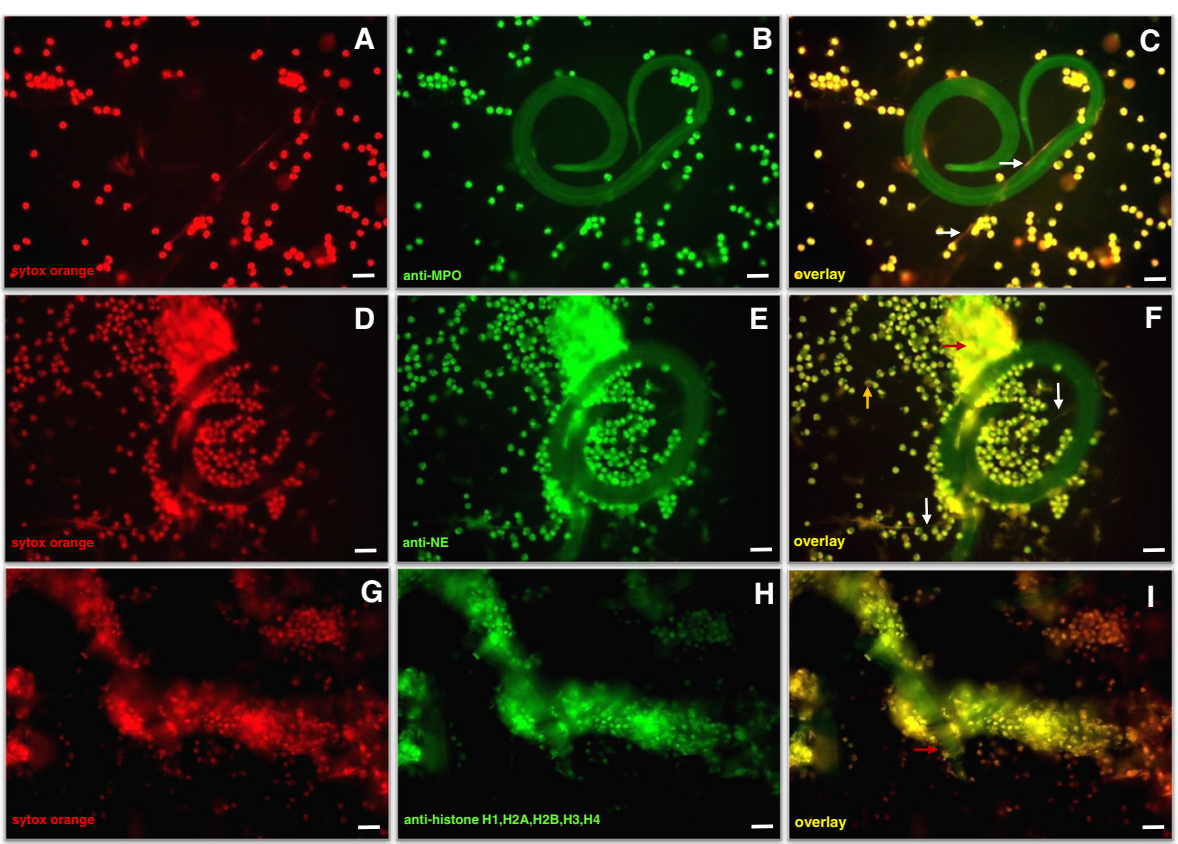

Fig. 2 Co-localization of exracellular DNA with histones, MPO and NE in H. contortus-triggered NET structures. Co-cultures of bovine PMN with H. contortus $L_{3}$ were fixed, permeabilized, stained for DNA using Sytox Orange and probed for histones using anti-histone H1, H2A/H2B, H3, H4 monoclonal antibodies, for MPO using anti-MPO and for NE using anti-NE antibodies. $\mathbf{a}, \mathbf{d}, \mathbf{g}$ : Extracellular DNA stained with Sytox Orange (red). $\mathbf{b}, \mathbf{e}, \mathbf{h}$ : MPO, NE and histones staining within NET structures (green), respectively. $\mathbf{c}, \mathbf{f}$, i: Overlay of NET-DNA with MPO, NE, and histones respectively. Note that distinct forms of NETs were observed in fluorescence microscopy: sprNET (c, f white arrows), aggNET (f red arrow) diffNET (f $\mathbf{f}$ yellow arrow) and clasp-like NET (i red arrow). Photomicrographs represent exemplary images of 3 independent experiments 


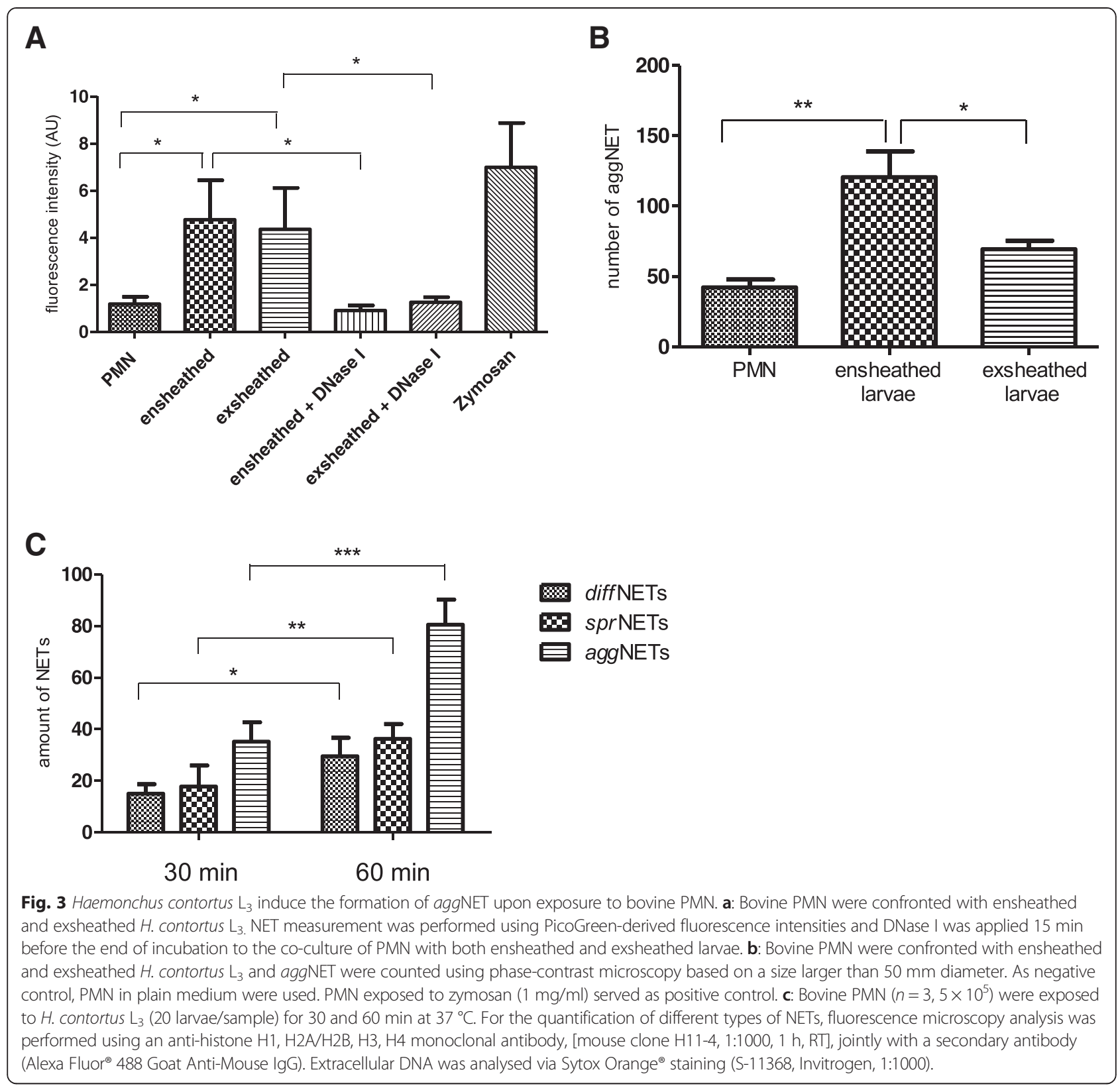

increased ( $p \leq 0.001$, Fig. 3c). Interestingly, at both time points predominantly aggNET were detected more frequently. Most probably, sprNET and diffNET are stages of the same process being aggNETs the latest stage. The difference of diffNET and sprNET formation along the two time points exhibited a significant increased $(p \leq 0.05$ and $p \leq 0.01$, respectively; Fig. $3 c$ ) showing overall a clear timedependent pattern.

\section{Haemonchus contortus $\mathrm{L}_{3}$-triggered NETosis occurs irrespective of parasite viability or $\mathrm{pH}$ environmental conditions}

In order to analyse the role of $H$. contortus $\mathrm{L}_{3}$ viability and integrity in parasite-induced NETosis, either viable or heat- inactivated larvae were used for NET induction. The quantitative assessment of NETs revealed that both preparations equally induced NETosis (viable and heat-inactivated larvae vs. control: both $p \leq 0.05$; Fig. 4a), i.e. the viability or integrity of larvae is irrelevant for NET induction.

Given that, following oral infection, ensheathed $\mathrm{L}_{3}$ of $H$. contortus reach the acidic environment of the abomasum and, after exsheathment, infect abomasal glands, we additionally tested whether NETosis also occurs in acidic conditions by testing different $\mathrm{pH}$ ranges $(2.0,2.5,3.0$ and 3.5) of the culture medium. Overall, even under low acidic conditions NETs were significantly formed in response to $H$. contortus $\mathrm{L}_{3}$ (pH 2.0 vs. control: $p \leq 0.05$; $\mathrm{pH} 2.5$ vs. control: $p \leq 0.05$; $\mathrm{pH} 3$ vs. control: $p \leq 0.05$; 


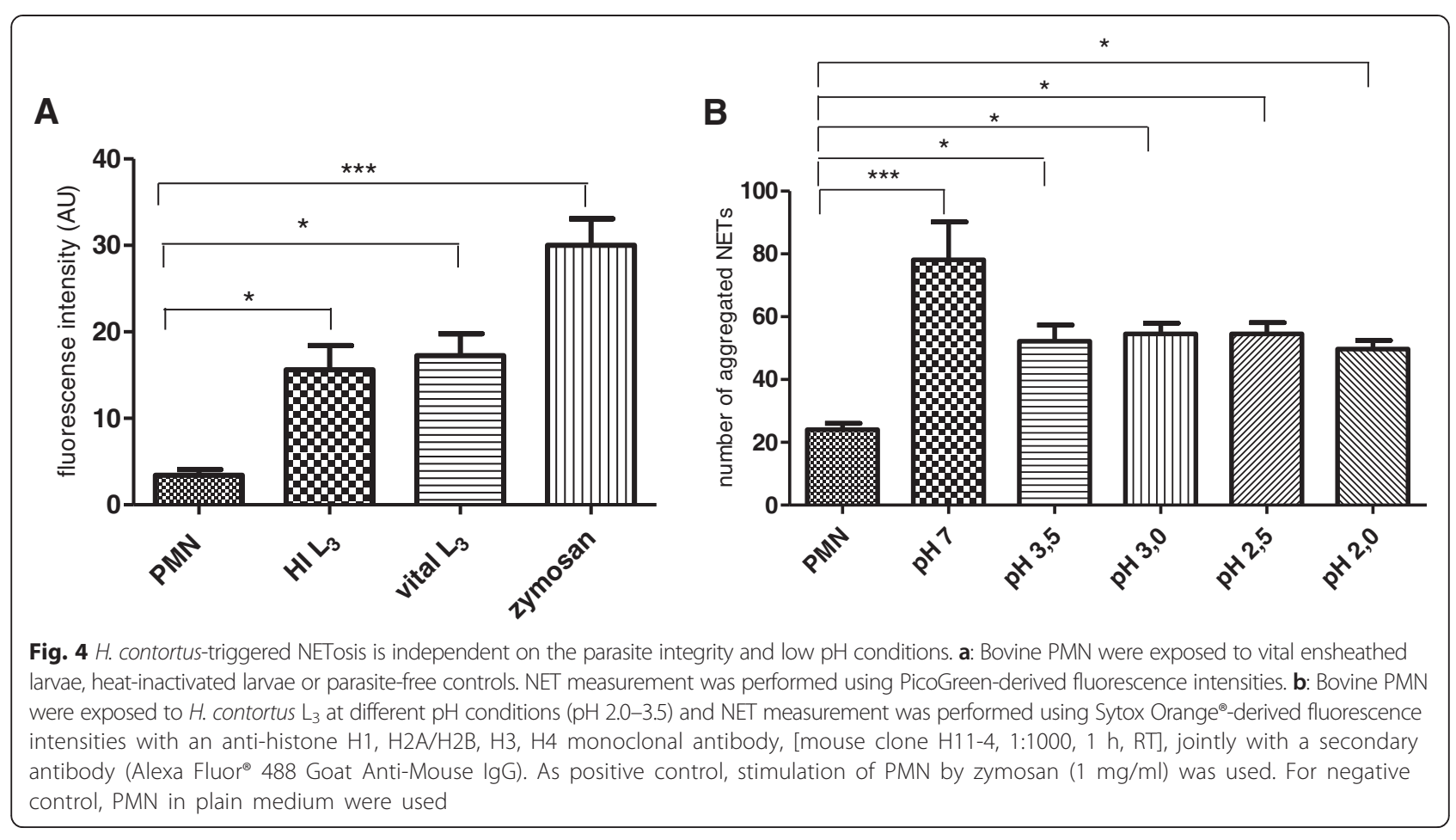

pH 3.5 vs. control: $p \leq 0.05$, Fig. 4b). However, at pH 7 a significantly stronger reaction was observed compared to the negative control $(p \leq 0.001)$ and lowering of the $\mathrm{pH}$ to 2.0 led to a reduction of NET formation of approximately $30 \%$ when compared to pH 7 (Fig. 4b).

NETs entrap Haemonchus contortus $L_{3}$ in a time-dependent and non-lethal manner

In order to evaluate time dependency of $H$. contortusmediated NET entrapment, bovine PMN were incubated with $H$. contortus for different time spans. As a result, significant differences were observed between 30 and $60 \mathrm{~min}$ of exposure ( $p \leq 0.01$, Fig. 5) leading to $46.9 \%$ and $75.8 \%$ entrapment of larvae, respectively, indicating that this process is time-dependent.

To elucidate whether $H$. contortus $\mathrm{L}_{3}$ may be killed by NETs, parasite survival was evaluated by microscopic measures (motility + trypan blue exclusion test) following long-term PMN exposure. Overall, $H$. contortus larvae appeared reduced in their motility since only $80 \%$ of the larvae displayed active motility compared to $100 \%$ in non-exposed control larvae (data not shown). However, trypan blue exclusion test neither revealed damage of the larval cuticle nor trypan blue up-take indicating that the larvae were still alive after exposure to NETs.

\section{Haemonchus contortus-triggered NET formation is NADPH} oxidase and MPO-dependent

To elucidate the role of different NET-associated molecules in $H$. contortus $\mathrm{L}_{3}$-triggered NETosis, inhibition experiments were performed using blockers of NADPH oxidase (DPI), MPO (ABAH) and NE (CMK). Pretreatments of PMN with DPI, CMK and ABAH resulted in a significant reduction of parasite-induced NET formation when compared with controls lacking inhibitors $(p \leq 0.05$; Fig. 6), indicating a key role of NADPH oxidase, NE and $\mathrm{MPO}$ in $H$. contortus $\mathrm{L}_{3}$-mediated NETosis.

\section{Haemonchus contortus $\mathrm{L}_{3}$ induce eosinophil-derived ETs}

Ovine eosinophils were isolated at a purity of $30 \%$ as demonstrated in Diff-Quick-stained cytospins (Fig. 7a). Given that the proportion of eosinophils in these preparations was not high enough to perform reliable quantitative assays on ETosis and further enrichment attempts failed, we made attempts to detect ET formation microscopically. Following exposure to $H$. contortus $\mathrm{L}_{3}$, several eosinophils (indicated by their red granules) were found in direct contact to the larvae (Fig. 7b). In addition, some of these clearly extruded PicoGreen ${ }^{\bullet}$-positive DNA onto the larval surface indicating that these cells performed ETosis (Fig. 7b).

\section{Discussion}

The present study delivers first evidence on the release of ETs as part of the early innate immune response of PMN and eosinophils to third stage larvae of the nematode $H$. contortus, an important and pathogenic species in the global veterinarian sector $[6,48-50]$. NETs mainly consist of chromatin, nuclear histones ( $\mathrm{H} 1, \mathrm{H} 2 \mathrm{~A} / \mathrm{H} 2 \mathrm{~B}$, $\mathrm{H} 3, \mathrm{H} 4)$ and granular components, such as NE, MPO, 

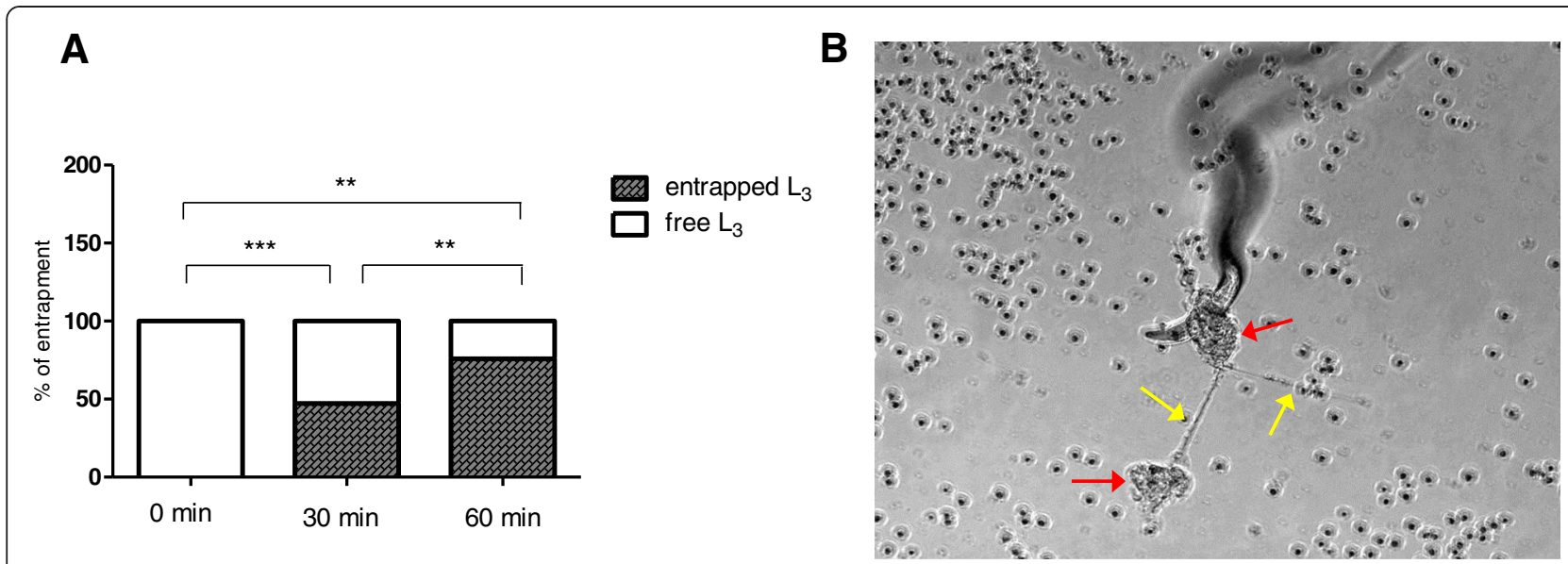

Fig. 5 Entrapment of $H$. contortus $L_{3}$ within NET structures is time-dependent. a: Bovine PMN were co-cultured with $H$. contortus $L_{3}$. Entrapped larvae were counted microscopically under different time points. $\mathbf{b}$ : Phase contrast image showing the anterior part of a $L_{3}$ being entrapped in NET structures. Red arrows: aggNET. Yellow arrows: anchor-like effect of fine NET structures mainly originating from aggNETs

lactoferrin and gelatinase $[16,51,52]$. We here confirmed the typical characteristics of NETs by co-localization experiments on extracellular DNA and histones, NE and MPO. Furthermore, functional inhibition experiments proved the relevance of NADPH oxidase, NE and MPO in $H$. contortus-induced NETosis since treatments with specific inhibitors significantly reduced parasite-triggered NET formation. This is in accordance to other reports on parasite-induced NET formation [30, 31, 39, 40, 45, 53].

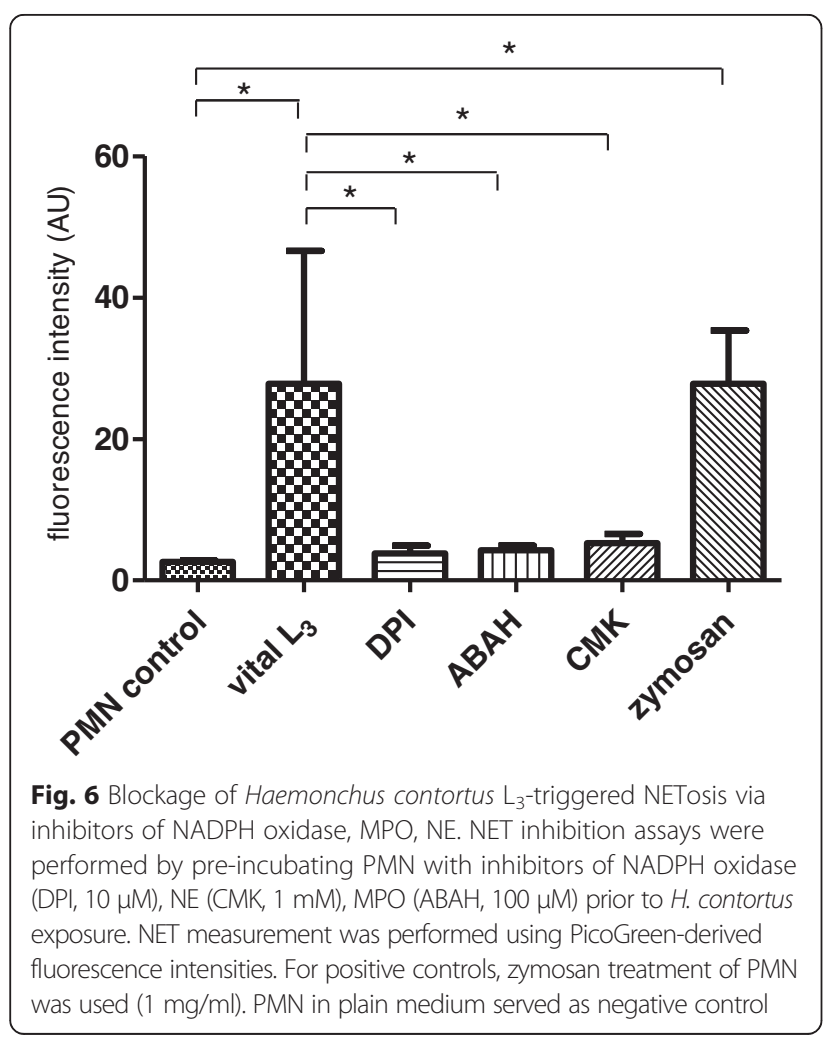

During haemonchosis, infective $H$. contortus $\mathrm{L}_{3}$ must actively migrate through the lumen of the reticulum, the rumen and the abomasum in order to infect gastric glands of the latter organ. By performing this migration, $H$. contortus larvae become potential targets of leucocytes of the innate immune system, which are known to actively migrate into the intestinal lumen [54-57]. This encounter drives the initiation of innate immune reactions as previously demonstrated by O'Connell et al. [58] showing a CXCR2/IL-17 dependent neutrophil recruitment in response to nematode infections. Referring to the infection biology of $H$. contortus, it has to be considered that exsheathed larvae will reside within the abomasum under low $\mathrm{pH}$ conditions. However, the current data on pH-dependent parasite-triggered NET formation clearly indicate that NETosis also occurs in these acidic conditions.

So far, several reports exist on protozoan-induced NET formation [31, 35-40, 45, 53, 59] whilst only few data are available on metazoan-triggered NETosis (for overview see Hermosilla et al., [26]). As such, S. japonicum was recently identified as NET-inducer in vitro and in vivo [41]. Another report demonstrated the capability of the nematode Strongyloides stercoralis to trigger NETosis [15]. In agreement with the current study, a NET-based ensnarement of $S$. stercoralis larvae was also demonstrated [15].

As an interesting feature, observed in this study was the release of different types of NETs, i.e. of diffNETs, sprNETs and aggNETs upon contact with $H$. contortus larvae, with all of them promoting a time-dependent ensnarement of the larvae. Given that no parasite killing was observed even within a prolonged period of $24 \mathrm{~h}$, the tight immobilization of larvae appears as the key mechanism of $H$. contortus-triggered NET formation. 

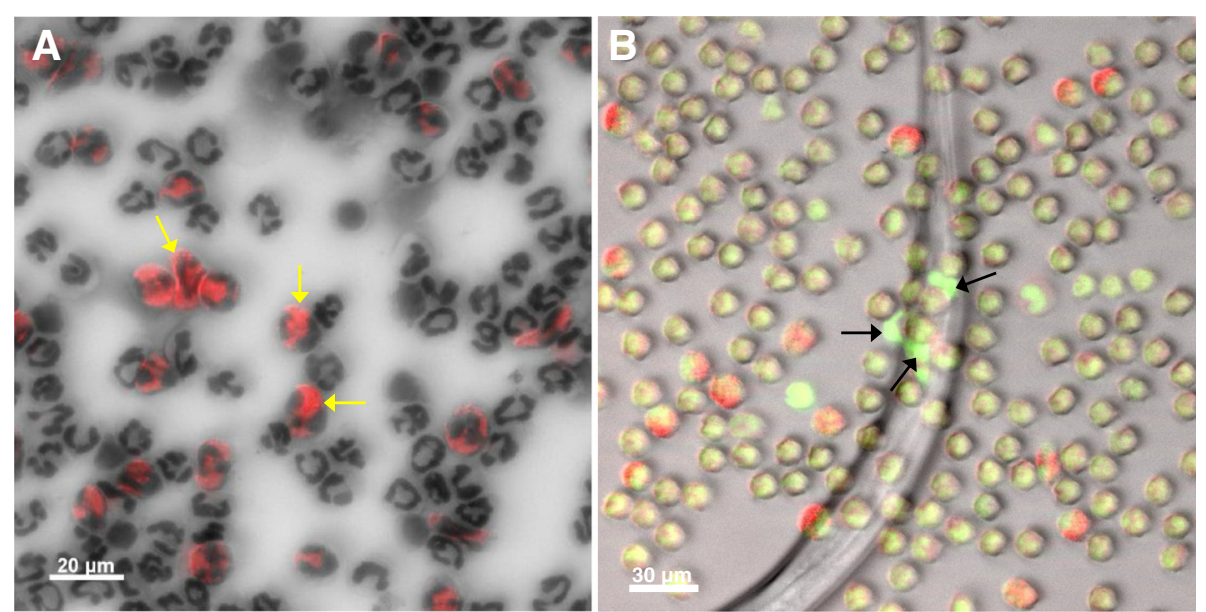

Fig. 7 Haemonchus contortus $L_{3}$ induce ETs in ovine eosinophils. a: Eosinophil population from ovine blood showing $30 \%$ of enrichment as detected in Diff-Quick-stained cytospin preparations. b: Eosinophil-derived ETs being released towards an $H$. contortus $\mathrm{L}_{3}$ [see co-localization of eosin granules (red) with eosinophil-derived extracellular DNA (green)]

Therefore, we hypothesize that even though NETs themselves do not appear capable of killing $H$. contortus larvae, they may entrap larvae and prevent the active migration into the gastric glands of the abomasum and expose them to other leucocytes.

It is intriguing that parasite-mediated NET induction proved to be independent of the ensheathment status of the larvae, since both, ensheathed and exsheathed larvae equally triggered NET formation. So far, it is unclear how PMN recognize the larvae in terms of NETosis and which parasite-derived molecules are involved this process. However, the data on ex- and ensheathed larvae suggest that the molecules responsible are present on the cuticle and the surface of the larval body. In contrast, the fact that ensheathed larvae induce aggNETs more prominently than exsheathed ones also indicates the involvement of different parasite-derived triggers. However, PMN-derived sensing of the larvae may also be a matter of size since Branzk et al. [60] reported on the ability of PMN to distinguish between small and large sized pathogens and to selectively release NETs in response to large pathogens [60]. In addition, other physical properties of particles such as shape and rigidity can influence the response of phagocytes [61]. This would explain the induction of NET formation by large metazoan parasites such as $H$. contortus larvae, and makes biological sense as phagocytosis is probably ineffective against large multicellular pathogens. We hypothesize that a function of PMN-released aggNETs may be to prevent proper larval exsheathment in vivo. A blockage of larval exsheathment would abrogate parasite infections and thereby tremendously affect the outcome of disease. We also consider that ETs might serve to localize anthelmintic products of neutrophils and eosinophils in the near vicinity of parasites. As such, neurotoxin (eosinophil-derived neurotoxin or EDN) released by eosinophils would be more effective if localized near the parasite. The release of EDN may restrict motility of the larvae thereby preventing the shedding process and allowing adhering eosinophils to discharge their toxic granule content directly on the larval surface [20,62]. This might serve to enhance the effects of these molecules impacting on larval motility. Moreover, parasite killing may be more likely to succeed if these anthelmintic molecules remain concentrated in the near vicinity of the parasite. Furthermore, ETosis is not a unique feature of PMN [63] but is also attributed to other immune cells, such as macrophages [64], mast cells $[27,65]$, monocytes [30, 31] and eosinophils [29, 66]. In accordance, we provide evidence on $H$. contortus-induced eosinophil-derived ETs (EETs). Meanwhile, in vivo studies with the nematode Nippostrongylus brasiliensis [62] demonstrate the formation of similar 'ET like entities' in presence of eosinophil-rich leucocytes and complement. In this model, larvae recovered from skin air pouches were aggregated into large clumps that included numerous leucocytes. Whilst many larvae eventually escaped from these aggregations, in mice with pre-existing eosinophilia, subsequent larval migration to the lungs was impaired [62]. This suggests that ET, leucocytes, and especially eosinophils, may be involved in damaging larvae in the very earliest stages of infection, such that the parasites are then more susceptible to entrapment and killing elsewhere in the pre-lung phases of migration through the host [62]. This may be of high relevance since eosinophils have been shown to adhere to $H$. contortus larvae both in vitro and in vivo and to efficiently kill these larvae [20]. Interestingly, Yousefi et al. [29] demonstrated that eosinophils release EETs of mitochondrial origin without undergoing cell death. However, whether $H$. contortustriggered EETs also originating from mitochondria remains 
to be elucidated in the future. Nonetheless, our data strongly suggest EETs as an additional effector mechanism of eosinophils against $H$. contortus.

\section{Conclusion}

Overall, we postulate that NETs and EETs may limit the establishment of $H$. contortus within the definitive host by immobilizing the larvae and hampering them from migration to the site of infection. NETs and EETs may further facilitate the exposure of entrapped large sized parasites to other immunocompetent cells, such as monocytes and macrophages exhibiting larvicidal effects. In consequence, $H$. contortus-mediated ET formation will also have an impact on the in vivo situation and influence the outcome of the disease.

\section{Additional file}

Additional file 1: Parasite entrapment by aggNETs and the anchor-like effect of fine NET structures (sprNETs) derived from aggNETs. (AVl $474248 \mathrm{~kb}$ )

Additional file 2: Significant reduction in larval motility due to an anchor-like effect of fine NETs originated from aggNETs.

(AVI 398927 kb)

\section{Competing interests}

The authors declare that they have no competing interests.

\section{Authors' contributions}

TMC and MCRR performed the NET quantification and in vitro experiments including Haemonchus contortus exsheathment and visualization of NETs. TMC and MCRR also participated in data analysis, design and coordination of the study and preparation of the manuscript. LMRS performed in vitro experiments including aggregated NET quantification and contributed in design and data analysis. UG and GM participated in preparation of samples and performance of scanning electron microscopy analysis. TMC, LMRS and TNM contributed in the isolation of eosinophils and ETs visualization in fluorescence microscopy. TNM also contributed in the correction of the manuscript. $\mathrm{CH}$ and AT participated in study design and coordination, data analysis and revision of the manuscript. In addition they contributed to blood collection for isolation of bovine PMN. All authors read and approved the final manuscript.

\section{Acknowledgements}

We would like to thank K. Becker and A. Mohr for their technical assistance in larval collection and C. Bauer for the kind donation of the Haemonchus contortus strain. TNM receives funding from the Scottish Government.

\section{Author details}

${ }^{1}$ Institute of Parasitology, Justus Liebig University Giessen, Giessen, Germany. ${ }^{2}$ Faculty of Veterinary Medicine and Zootechny, Autonomous University of Sinaloa, Culiacán, Mexico. ${ }^{3}$ Institute of Anatomy and Cell Biology, Justus Liebig University, Giessen, Germany. ${ }^{4}$ Moredun Research Institute, Penicuik, UK.

Received: 26 August 2015 Accepted: 19 November 2015 Published online: 26 November 2015

\section{References}

1. Mugambi JM, Bain RK, Wanyangu SW, Ihiga MA, Duncan JL, Murray M, Stear MJ.Resistance of four sheep breeds to natural and subsequent artificial Haemonchus contortus infection. Vet Parasitol. 1997;69:265-73.

2. Roberts F, Newton-Turner H, McKevett M. On the specific distinctness of the ovine and bovine 'Strains' of Haemonchus contortus (Rudolphi) Cobb Nematoda: Trichostronglidae. Aust J Zool. 1954;2:275-95.
3. Bemrick W, Emerick R, Shumard R, Pope A, Phillips P. The effect of bleeding versus previous infection on the resistance of lambs to subsequent infections of Haemonchus contortus. J Anim Sci. 1958;17:363-7.

4. Allen R, Schad G, Samson K. Experimental cross-transmission of two strains of Haemonchus from wild ruminants to domestic sheep, with observations on their pathogenecity as compared with Haemonchus from domestic sheep. J Parasitol. 1958;44:26.

5. Riffkin G, Dobson C. Predicting resistance of sheep to Haemonchus contortus infections. Vet Parasitol. 1979;5:365-78.

6. Tan TK, Panchadcharam C, Low VL, Lee SC, Ngui R, Sharma RSK, et al. Co-infection of Haemonchus contortus and Trichostrongylus spp. among livestock in Malaysia as revealed by amplification and sequencing of the internal transcribed spacer II DNA region. Vet Res. 2014;10:38.

7. Cordero del Campillo M, Rojo Vazquez FA, Martínez Fernandez AR. Parasitología Veterinaria, McGraw-Hill, Madrid, España, 1999.

8. Sackett D, Holmes PH, Abbott K, Jephcott S, Barber M. Assessing the economic cost of endemic disease on the profitability of Australian beef cattle and sheep producers AHW.087 Report, Meat and Livestock Australia, North Sydney. 2006, 119.

9. Balic A, Bowles VM, Meeusen ENT. Cellular profiles in the abomasal mucosa and lymph node during primary infection with Haemonchus contortus in sheep. Vet Immunol Immunopath. 2000;75:109-20.

10. Balic A, Cunningham CP, Meeusen ENT. Eosinophil interactions with Haemonchus contortus larvae in the ovine gastrointestinal tract. Parasit Immunol. 2006;28:107-15.

11. Lacroux C, Nguyen TH, Andreoletti O, Prevot F, Grisez C, Ergeaud JPB, et al Haemonchus contortus (Nematoda: Trichostrongylidae) infection in lambs elicits an unequivocal Th2 immune response. Vet Res. 2006;37:607-22.

12. Meeusen ENT, Balic A, Bowles V. Cells, cytokines and other molecules associated with rejection of gastrointestinal nematode parasites. Vet Immunol Immunopathol. 2005;108:121-5.

13. Miller HRP. Prospects for the immunological control of ruminant gastrointestinal nematodes: natural immunity, can it be harnessed? Int J Parasitol. 1996;26(8):801-11.

14. Meeusen ENT, Balic A. Do eosinophils have a role in the killing of helminth parasites? Parasitol Today. 2000;16(3):95-101

15. Bonne-Année S, Kerepesi LA, Hess JA, Wesolowski J, Paumet F, Lok JB, et al. Extracellular traps are associated with human and mouse neutrophil and macrophage mediated killing of larval Strongyloides stercoralis. Microbes Infect. 2014;16(6):502-11.

16. Brinkmann V, Reichard U, Goosmann C, Fauler B, Uhlemann Y, Weiss DS, et al. Neutrophil extracellular traps kill bacteria. Sci. 2004;303:1532-5.

17. Urban CF, Ermert D, Schmid M, Abu-Abed U, Goosmann C, Nacken W, et al. Neutrophil extracellular traps contain calprotectin, a cytosolic protein complex involved in host defense against Candida albicans. PLoS Pathog. 2009;5:10.

18. Brinkmann $V$, Zychlinsky A. Neutrophil extracellular traps: is immunity the second function of chromatin? J Cell Biol. 2012;198:773-83.

19. Hahn S, Giaglis S, Chowdhury CS, Hosli I, Hasler P. Modulation of neutrophil NETosis: interplay between infectious agents and underlying host physiology. Semin Immunopathol. 2013;35:439-53.

20. Rainbird MA, McMillan D, Meeusen ENT. Eosinophil-mediated killing of Haemonchus contortus larvae: effect of eosinophil activation and role of antibody, complement and interleukin-5. Paras Immunol. 1998;20:93-103.

21. Terefe G, Lacroux C, Prévot F, Grisez C, Bergeaud JP, Bleuart C, et al. Eosinophils in Haemonchus contortus-infected resistant and susceptible breeds of sheep: abomasal tissue recruitment and in vitro functional state. Vet Parasitol. 2009;165(1-2):161-4.

22. Rotman HL, Yutanawiboonchai W, Brigandi RA, Leon O, Gleich GJ, Nolan TJ, et al. Strongyloides stercoralis: eosinophil-dependent immune-mediated killing of third stage larvae in BALB/CByJ mice. Exp Parasitol. 1996;82:267-78.

23. Folkard SG, Hogarth PJ, Taylor MJ, Bianco AE. Eosinophils are the major effector cells of immunity to microfilariae in a mouse model of onchocerciasis. Parasitol. 1996;112:323-9.

24. Grove DI, Mahmoud AA, Warren KS. Eosinophils and resistence to Trichinella spiralis. J Exp Med. 1977;145:755-9.

25. Gleich GJ, Lammas DA, Herlich $\mathrm{H}$. The efffect of antiserum to eosinophils on susceptibility and aquired immunity of the guineapig to Trichostrongylus colubriformis. Immunol. 1977;37:373-880.

26. Hermosilla C, Muñoz Caro T, Silva LMR, Ruiz A, Taubert A. The intriguing host innate immune response: novel anti-parasitic defence by neutrophil extracellular traps. Parasitol. 2014;141(11):1489-98. doi:10.1017/S0031182014000316c 
27. von Köckritz-Blickwede M, Goldmann O, Thulin P, Heinemann K, Norrby-Teglund A, Rohde $M$, et al. Phagocytosis-independent antimicrobial activity of mast cells by means of extracellular trap formation. Blood. 2008;111:3070-80

28. Chow OA, von Kockritz-Blickwede M, Bright AT, Hensler ME, Zinkernagel AS, Cogen $A L$, et al. Statins enhance formation of phagocyte extracellular traps. Cell Host Microbe. 2010;8:445-54.

29. Yousefi S, Gold JA, Andina N, Lee JJ, Kelly AM, Kozlowski E, et al. Catapult-like release of mitochondrial DNA by eosinophils contributes to antibacterial defense. Nat Med. 2008;14:949-53.

30. Muñoz-Caro T, Silva LM, Ritter C, Taubert A, Hermosilla C. Besnoitia besnoiti tachyzoites induce monocyte extracellular trap formation. Parasitol Res. 2014;113(11):4189-97.

31. Reichel M, Muñoz-Caro T, Sanchez-Contreras G, Rubio-García A, Magdowski G, Gärtner U, et al. Harbour seal (phoca vitulina) PMN and monocytes release extracellular traps to capture the apicomplexan parasite Toxoplasma gondii. Dev Comp Immunol. 2015;50:106-15

32. Fuchs TA, Abed U, Goosmann C, Hurwitz R, Schulze I, Wahn V, et al. Novel cell death program leads to neutrophil extracellular traps. J Cell Biol. 2007;176(2):231-41.

33. Aulik NA, Hellenbrand KM, Klos H, Czuprynski CJ. Mannheimia haemolytica and its leukotoxin cause neutrophil extracellular trap formation by bovine neutrophils. Infect Immun. 2010;78(11):4454-66.

34. Yipp BG, Petri B, Salina D, Jenne CN, Scott BN, Zbytnuik LD, et al. Infectioninduced NETosis is a dynamic process involving neutrophil multitasking in vivo. Nat Med. 2012;18(9):1386-93.

35. Baker VS, Imade GE, Molta NB, Tawde P, Pam SD, Obadofin MO, et al. Cytokine-associated neutrophil extracellular traps and antinuclear antibodies in Plasmodium falciparum infected children under six years of age. Malar J. 2008;29(7):41.

36. Guimarães-Costa AB, Nascimento MT, Froment GS, Soares RP, Morgado FN, Conceicao-Silva F, et al. Leishmania amazonensis promastigotes induce and are killed by neutrophil extracellular traps. Proc Natl Acad Sci. 2009;106:6748-53.

37. Behrendt JH, Ruiz A, Zahner H, Taubert A, Hermosilla C. Neutrophi extracellular trap formation as innate immune reactions against the apicomplexan parasite Eimeria bovis. Vet Immunol Immunopathol. 2010;133:1-8.

38. Abi Abdallah DS, Lin C, Ball CJ, King MR, Duhamel GE, Denkers EY. Toxoplasma gondii triggers release of human and mouse neutrophil extracellular traps. Infect Immun. 2012;80:768-77.

39. Muñoz-Caro T, Hermosilla C, Silva MRL, Cortes H, Taubert A. Neutrophil extracellular traps as innate immune reaction against the emerging apicomplexan parasite Besnoitia besnoiti. PloS One. 2014;11;9(3):e91415.

40. Silva LMR, Muñoz Caro T, Rüdiger G, Vila-Viçosa M.J.M, Cortes H, Hermosilla T, Taubert, A. The apicomplexan parasite Eimeria arloingi induces caprine neutrophil extracellular traps. Parasitol Res. 2014. doi:10.1007/s00436-014-3939-0

41. Chuah C, Jones MK, Burke ML, Owen HC, Anthony BJ, Mcmanus DP, et al. Spatial and temporal transcriptomics of Schistosoma japonicum-induced hepatic granuloma formation reveals novel roles for neutrophils. J Leukoc Biol. 2013;94:353-65.

42. Roberts FHS, O'Sullivan PJ. Methods for egg counting and larval cultures for strongylesinfesting the gastrointestinal tract of cattle. Aust J Agric Res. 1950;1:99-102.

43. Martinelli S, Urosevic M, Daryadel A, Oberholzer PA, Baumann C, Fey MF, et al. Induction of genes mediating interferon-dependent extracellular trap formation during neutrophil differentiation. J Biol Chem. 2004;279:44123-32.

44. Lippolis JD, Reinhardt TA, Goff JP, Horst RL. Neutrophil extracellular trap formation by bovine neutrophils is not inhibited by milk. Vet Immunol Immunopathol. 2006;15:113(1-2):248-55.

45. Muñoz-Caro T, Mena Huertas SJ, Conejeros I, Alarcón P, Hidalgo MA, Burgos RA, et al. Eimeria bovis-triggered neutrophil extracellular trap formation is CD11b-, ERK 1/2-, p38-MAP kinase- and SOCE-dependent. Vet Res. 2015;46:23.

46. Scapinello S, Brooks AS, MacInnes Jl, Hammermueller J, Clark ME, Caswell JL. Bactericidal activity of porcine neutrophil secretions. Vet Immunol Immunopathol. 2011;139:113-8.

47. Schauer C, Janko C, Munoz LE, Zhao Y, Kienhöfer D, Frey B, et al Aggregated neutrophil extracellular traps limit inflammation by degrading cytokines and chemokines. Nat Med. 2014;20(5):511-7.

48. Prestwood AK, Kellogg FE, Pursglove SR. Hayes FA Helminthic parasitism among intermingling insular populations of white-tailed deer, feral cattle and feral swine. J Am Vet Med Assoc. 1975;166(1):787-90.
49. Mckenzie ME, Davidson WR. Helminths parasites of intermingling axis deer, wild swine and domestic cattle from the island of Molokai, Hawaii. J Wild Dis. 1989;25(2):253-7

50. Getachew T, Dorchies P, Jacquiet P. Trends and challenges in the effective and sustainable control of Haemonchus contortus infection in sheep. Parasite. 2007;14:3-14.

51. Wartha F, Beiter K, Albiger B, Fernebro J, Zychlinsky A, Normark S, et al. Capsule and D-alanylated lipoteichoic acids protect Streptococcus pneumoniae against neutrophil extracellular traps. Cell Microbiol. 2007;9:1162-71.

52. Papayannopoulos V, Metzler KD, Hakkim A, Zychlinsky A. Neutrophil elastase and myeloperoxidase regulate the formation of neutrophil extracellular traps. J Cell Biol. 2010;191(3):677-91.

53. Muñoz-Caro T, Lendner M, Daugschies A, Hermosilla C, Taubert A. NADPH oxidase, MPO, NE, ERK1/2, p38 MAPK and $\mathrm{Ca}(2+)$ influx are essential for Cryptosporidium parvum-induced NET formation. Dev Comp Immunol. 2015. doi:10.1016/j.dci.2015.05.007

54. Brazil JC et al. alpha3/4 Fucosyltransferase 3-dependent synthesis of Sialyl Lewis A on CD44 variant containing exon 6 mediates polymorphonuclear leukocyte detachment from intestinal epithelium during transepithelial migration. J Immunol. 2013;191(9):4804-17.

55. Seper A, Hosseinzadeh A, Gorkiewicz G, Lichtenegger S, Roier S, Leitner DR et al. Vibrio cholerae evades neutrophil extracellular traps by the activity of two extracellular nucleases. PLoS Pathog. 2013;9:9.

56. Sumagin R, Robin AZ, Nusrat A, Parkos CA. Transmigrated neutrophils in the intestinal lumen engage ICAM-1 to regulate the epithelial barrier and neutrophil recruitment. Mucosal Immunol. 2013. doi:10.1038/mi.2013.106

57. Szabady RL, McCormick BA. Control of neutrophil inflammation at mucosal surfaces by secreted epithelial products. Front Immunol. 2013;4:220.

58. O'Connell AE, Redding KM, Hess JA, Lok JB, Nolan TJ, Abraham D. Soluble extract from the nematode Strongyloides stercoralis induces CXCR2 dependent/IL-17 independent neutrophil chemotaxis. Microbes Infect. 2011;13:536-44.

59. Gabriel C, McMaster WR, Girard D, Descoteaux A. Leishmania donovani promastigotes evade the antimicrobial activity of neutrophil extracellular traps. J Immunol. 2010;185(7):4319-27.

60. Branzk N, Lubojemska A, Hardison SE, Wang Q, Gutierrez MG, Brown GD, et al. Neutrophils sense microbe size and selectively release neutrophil extracellular traps in response to large pathogens. Nat Immunol. 2014;15(11):1017-25.

61. Wheeler ML, Underhill DM. Time to cast a larger net. Nat Immunol. 2014;15:1000-1. doi:10.1038/ni.3013.

62. Giacomin PR, Gordon DL, Botto M, Daha MR, Sanderson SD, Taylor SM, et al. The role of complement in innate, adaptive and eosinophil-dependent immunity to the nematode Nippostrongylus brasiliensis. Mol Immunol. 2008;45(2):446-55

63. Cheng OZ, Palaniyar N. NET balancing: a problem in inflammatory lung diseases. Front Immunol. 2013:4:1.

64. Hellenbrand KM, Forsythe KM, Rivera-Rivas JJ, Czuprynski CJ, Aulik NA. Histophilus somni causes extracellular trap formation by bovine neutrophils and macrophages. Microb Pathog. 2013;54:67-75.

65. Lin AM, Rubin CJ, Khandpur R, Wang JY, Riblett M, Yalavarthi S, et al. Mast cells and neutrophils release IL-17 through extracellular trap formation in psoriasis. J Immunol. 2011;187(1):490-500.

66. Dworski R, Simon HU, Hoskins A, Yousefi S. Eosinophil and neutrophil extracellular DNA traps in human allergic asthmatic airways. J Allergy Clin Immunol. 2011;127(5):1260-6.

\section{Submit your next manuscript to BioMed Central and we will help you at every step:}

- We accept pre-submission inquiries

- Our selector tool helps you to find the most relevant journal

- We provide round the clock customer support

- Convenient online submission

- Thorough peer review

- Inclusion in PubMed and all major indexing services

- Maximum visibility for your research

Submit your manuscript at www.biomedcentral.com/submit 